\title{
Percepção Materna Sobre Saúde Bucal: um Estudo em um Hospital de Referência do Estado da Paraíba
}

\author{
Maternal Perception on Oral Health: \\ a Study in a Reference Hospital in Paraíba State
}

\section{RESUMO}

Objetivo: Esta pesquisa avaliou a percepção das mães em relação aos cuidados com a sua saúde bucal e da criança nos primeiros anos de vida. Material e Métodos: Trata-se de um estudo transversal com abordagem quantitativa. Os dados foram obtidos mediante questionários aplicados às mães que frequentaram o serviço de Pediatria do Hospital Universitário Lauro Wanderley, Universidade Federal da Paraíba, João Pessoa, Paraíba, Brasil, no período de outubro de 2012 a março de 2013. Resultados: Das 60 mães com filhos de idade de 0 a 11 anos, $43 \%$ não receberam orientações sobre as doenças cárie e inflamação gengival, e não sabem o que pode causá-las ou como preveni-las. Relatam saber cuidar da saúde da sua boca, porém $56 \%$ delas não obteve orientação quanto aos cuidados bucais da criança,43\%não levaram seu filho ao dentista e citamsua ida quando sente dor, $38 \%$ das mãesacham a saúde da sua boca regular. As mães relataram escovar os dentes, uso de enxaguatório e fio dental como cuidados da saúde de sua boca e da criança, e $26 \%$ delas não souberam formular o que causa a cárie. Conclusão: As mães participantes da pesquisa atendidas na Pediatria do HULW possuem uma percepção de sua saúde bucal razoável.

\section{DESCRITORES}

Odontopediatria. Higiene Bucal. Educação em Saúde Bucal.

\begin{abstract}
Objective: This study evaluated the perception of mothers on their oral health care and of their child in the early years of life. Materials and Methods: This was a cross-sectional study with a quantitative approach. Data were collected through questionnaires submitted o mothers who attended the Lauro Wanderley University Hospital Pediatrics Service, Federal University of Paraíba, João Pessoa, Paraíba, Brazil, from October 2012 to March 2013. Results: Of the 60 mothers of children aged $0-11$ years, $43 \%$ did not receive guidance on caries and gingival inflammation, and did not have information about the disease etiology and preventive measures. The mothers reported knowing how to manage their own oral care, but $56 \%$ of them did not receivegui dance on the oral care of their children; $43 \%$ had not taken their children to the dentist yet - and reported doing so only when the child referred pain; $38 \%$ of the mothers considered their oral health as regular. The mothers reported brushing the teeth, using mouthwash and flossing as health care measures; $26 \%$ of them were unable to formulate what causes tooth decay. Conclusion: The survey participants showed a reasonable perception of their oral health. Investments in programs for oral health prevention and promotion in this referral hospital should be established to improve the knowledge and hygiene practices in oral health care.
\end{abstract}

\section{DESCRIPTORS}

Pediatric Dentistry. Oral Hygiene. Oral Health Education.

1 Mestranda em Ciências Odontológicas pela Universidade Federal da Paraíba (UFPB). João Pessoa. Paraíba. Brasil.

2 Doutor. Professor adjunto do curso de Odontologia da Universidade Federal de Campina Grande (UFCG). Patos. Paraíba. Brasil.

3 Doutora. Professora adjunta do curso de Odontologia da Universidade Federal da Paraíba (UFPB). João Pessoa. Paraíba. Brasil. 
A educação dos pais contribui para a manutenção da saúde bucal e uma redução significativa das doenças bucais em crianças na primeira infância ${ }^{1}$.A educação odontológica da mãe ou responsável é fator determinante para a futura saúde bucal da criança e a família serve como modelo auxiliando a criança a cuidar de seus dentes $^{2}$.

A mãe possui um importante papel dentro do núcleo familiar, tornando-se um ponto chave na prevenção de doenças, como condutora de hábitos alimentares da família e formadora de conceitos sobre saúde ${ }^{3}$. Condições de saúde gerais e bucais sofrem o impacto de fatores presentes desde o início da vida, a exemplo de níveis mais baixos de escolaridade materna, do baixo peso ao nascer e da má-nutrição. Esses fenômenos parecem incrementar o risco de cárie dental e são mais prevalentes em áreas de maior privação $0^{4,5}$.

Percepções dos pais sobre a saúde bucal de seus filhos podem influenciar as decisões de saúde bucal e os cuidados com a saúde ${ }^{6}$. As políticas de saúde devem investir em programas e serviços que objetivem a redução dos problemas de saúde bucal que acometem a população ${ }^{7}$.Os primeiros anos de vida são considerados os de maior risco para o desenvolvimento de cárie dental na dentição decídua ${ }^{8}$.Problemas de saúde bucal podem afetar a estrutura familiar devido ao impacto nas atividades de vida diária ${ }^{9}$.

Considerando a importância do conhecimento materno sobre a saúde bucal, tendo em vista que suas práticas podem evitar doenças de saúde pública como a cárie, essa pesquisa objetivou conhecer a percepção de mães em relação aos cuidados com a sua saúde bucal e a da criança nos primeiros anos de vida.

\section{MATERIAL E MÉTODOS}

O presente estudo foi aprovado pelo Comitê de Ética e Pesquisa em Seres Humanosdo Hospital Universitário Lauro Wanderley da Universidade Federal da Paraíba, João Pessoa, Paraíba, Brasil.

Trata-se de uma pesquisa transversal com abordagem quanti-qualitativa e técnica de observação direta extensiva.Os dados foram obtidos mediante entrevista com auxílio de questionários contendo 21 perguntas (figura 1) e aplicados às mães que frequentaram o serviço de Pediatria do Hospital Universitário Lauro Wanderley (HULW), Universidade Federal da Paraíba, no período de outubro de 2012 a março de 2013.0 questionário foi obtido e adaptado por meio dos estudos encontrados na literatura ${ }^{1,10-12}$.

O universo foi composto por todas as mães cujos filhos estavam sendo atendidos no período da pesquisa, no turno da manhã.Obteve-se uma amostrade60 mães (30\% do universo), as quais foram abordadas na sala de espera do serviço de Pediatria do HULW, selecionadas de acordo com a disponibilidade e o interesse em participar da pesquisa. As participantes receberam o Termo de Consentimento Livre e Esclarecido previamente à entrevista. Este abordava informações sobre a percepção e cuidados relativos à saúde bucal materna e da criança, como o que causa e previne a doença cárie, e o modo e a frequência da higienização da boca. Os dados foram computados no programa Microsoft Office Excel for Windows 2008, analisadospor meio de procedimento comparativo e estatístico de forma descritiva.

\section{RESULTADOS}

Os resultados referentes às respostas obtidas através dos questionários estão expostos na Tabela 1, Figura 2 e na Figura 3. As participantes relacionaram a escovação (95\%), uso do fio dental $(27,5 \%)$ para prevenir a cárie dentária, sendo que duas delas relataram a alimentação pobre em doces como forma de prevenção. Dentre as que possuíam bebês, uma participantese referiu ao uso de "pano ou fralda" para fazer a higiene da boca, enquanto outras cinco disseram não realizar nenhum cuidado quanto a esta higiene.

\section{DISCUSSÃO}

De acordo com os resultados desta pesquisa, $43 \%$ das mães relataram ter recebido orientações sobre os cuidados da saúde bucal dos seus filhos, corroborando com estudos relatados na literatura ${ }^{13,14} \mathrm{e}$ com uma pesquisa ${ }^{15}$ realizada no Hospital Universitário Alcides Carneiro - Campina 
Quadro 1: Questionário com roteiro pré-estabelecido destinado às mães sobre saúde bucal.

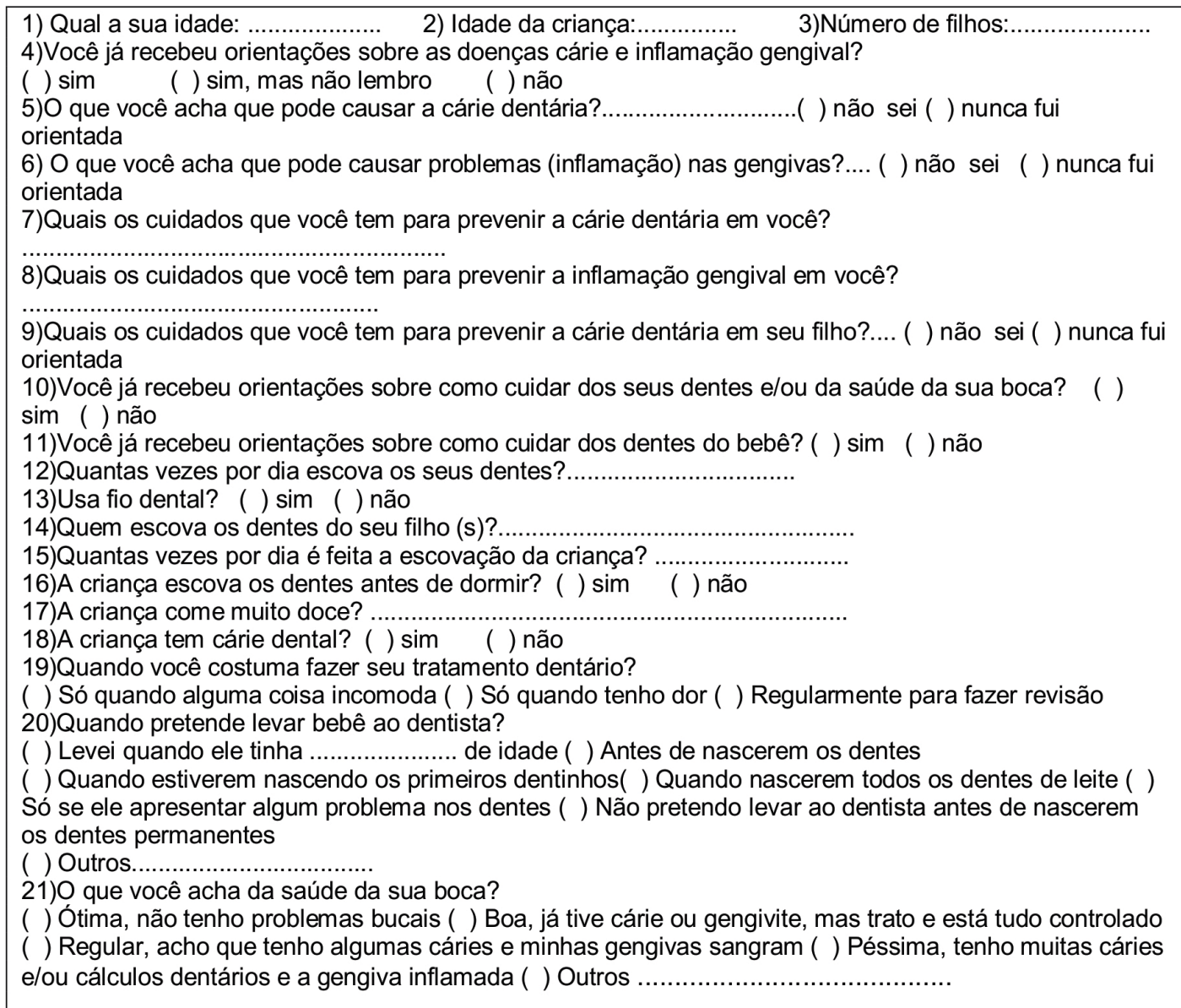

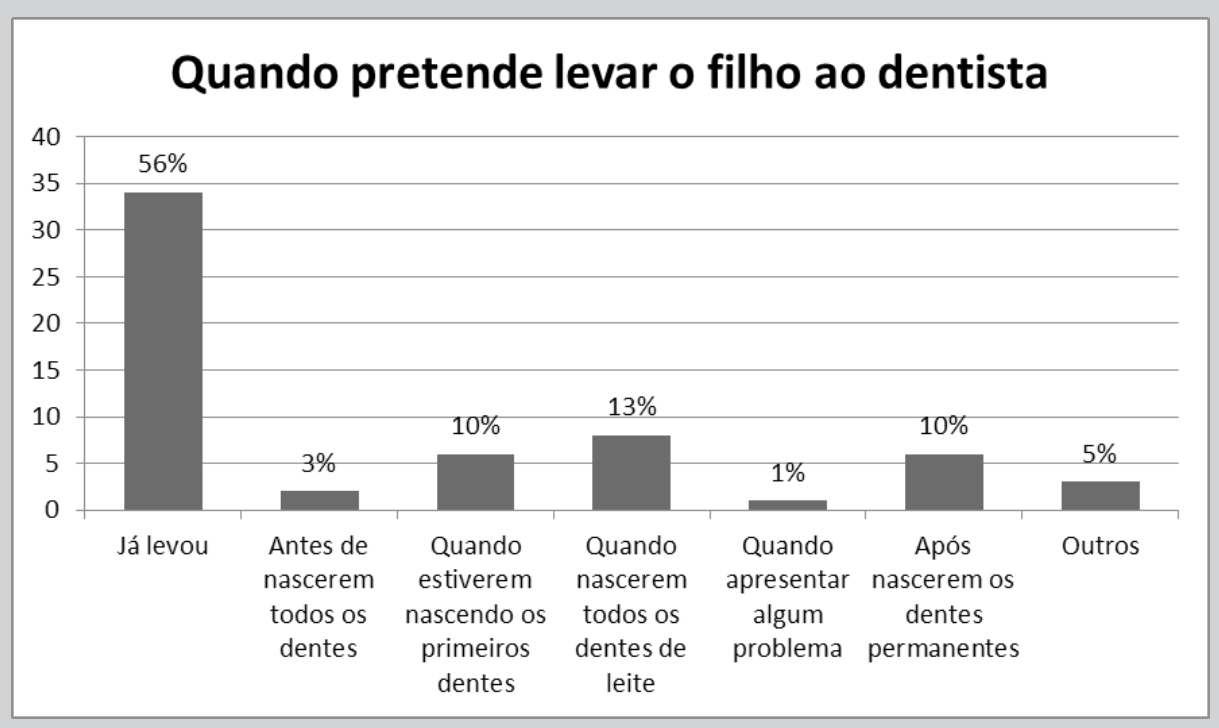

Figura 2- Respostas das mães referente a quando elas pretendem levar os filhos ao dentista. 


\section{Percepção das mães sobre sua saúde bucal}

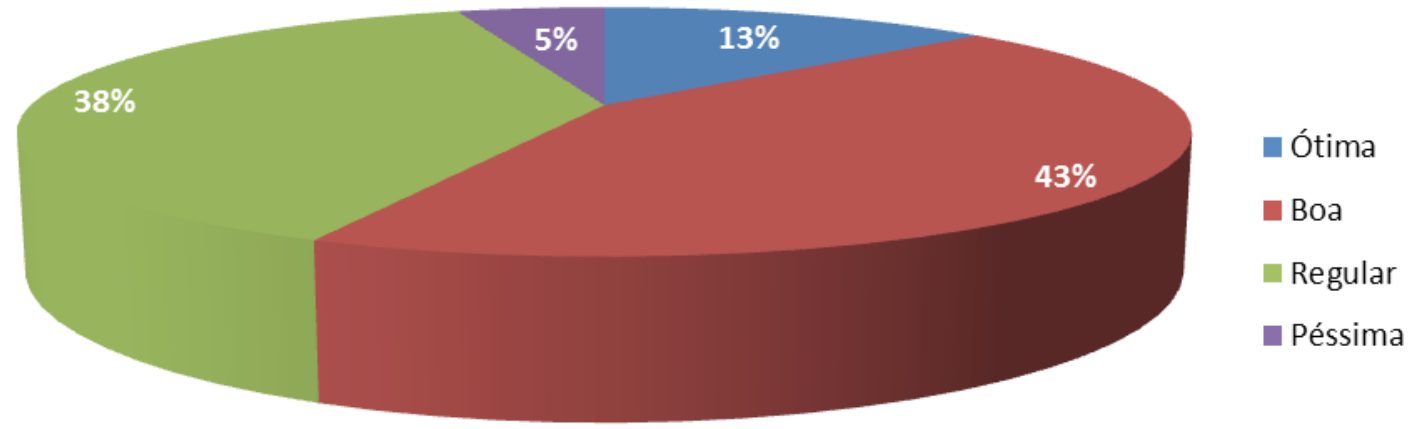

Figura 3- Respostas das mães sobre a questão: "O que você acha da saúde da sua boca"?

Grande, onde $32,5 \%$ das 80 mães entrevistadas também obtiveram esse conhecimento e contrastando com outros estudos ${ }^{16,17}$ que demonstraram que o maior percentual de entrevistadas receberam informações sobre saúde bucal. Nesses estudos, a população estudada (mães ou gestantes) pode ter obtido tal conhecimento através de participação em palestras de promoção de saúde. Destacandose assim, a importância de atividades de promoção e prevenção da saúde por parte dos profissionais.

Nesta pesquisa $56 \%$ das mães é a responsável por escovar os dentes da criança e $11 \%$ são os filhos que o fazem, sendo que $81 \%$ afirma que essa escovação é feita entre 2 a 3 vezes ao dia. Essesresultados mostraram semelhança com outro estudo ${ }^{12}$ que relatou que $81,3 \%$ são as mães que escovam e $17,8 \%$ as crianças, com $80 \%$ relatando essa mesma frequência de higienização. Outros estudos ${ }^{11,15}$ também demonstram essas frequências como as mais citadas.

Nesta pesquisa as mães citaram a escovação e a dieta como meio de maior prevenção da cárie corroborando com um estudo ${ }^{10}$ realizado na Maternidade do Instituto Cândida Vargas no município de João Pessoa - PB,o qual obteve 45\% de resposta de escovação e $20 \%$ de diminuição do açúcar como medida preventiva dessa doença.

Recomenda-se o período de seis meses de vida ideal para o atendimento odontológico ${ }^{18}$. No presente estudo, $56 \%$ das entrevistadasdisseram já ter levado seus filhos ao dentista e $10 \%$ das mães pretendem levar os filhos ao dentista quando estiverem nascendo os primeiros dentes. Estudos ${ }^{10,11,16,17}$ mostram que o maior percentual de entrevistadas afirma que a visita deve ser realizada no momento de nascimento dos dentes. A maioria dos pais leva seus filhos ao dentista por volta dos dois e três anos de idade, quando a dentição decídua já está completa e, em alguns casos, o processo carioso já está instalado ${ }^{19}$.

Quanto à autopercepção da saúde bucal, $81 \%$ das mães que participaram da pesquisa relataram que a sua condição bucal se encontra de boa a regular, corroborando com a literatura ${ }^{19-21}$.

De acordo com os resultados desse estudo, observa-se que as mães participantes da 
pesquisa apresentam uma grande carência de informações sobre o que causa ( $66 \%$ ) e $53 \%$ delas não sabem como prevenir a inflamação gengival. As pesquisas presentes na literatura são escassas em relação à percepção de mães sobre a gengivite.

Mesmo com os grandes avanços no acesso à Odontologia, as mães que frequentaram o serviço de Pediatria do Hospital Universitário Lauro Wanderley, mostraram carência de informação com relação aos cuidados da sua saúde bucal e de seus filhos. A necessidade de programas que visem a difusão de orientações quanto a questões de saúde pública como o que é a doença cárie e a gengivite, e suas formas de prevenção é muito importante. Pois dessa forma, as mães se tornam mais aptas aos cuidados com a sua saúde bucal e das crianças.

\section{CONCLUSÃO}

As mães participantes da pesquisa atendidas no setor de Pediatria do HULW possuem uma percepção razoável de sua saúde bucal.

\section{REFERÊNCIAS}

1. Simioni LRG, Comiotto MS, Rêgo DM. Percepções maternas sobre a saúde bucal de bebês: da informação à ação. RPG, Rev Pós-Grad. 2005;12(2):167-73.

2. Ferreira ARC, Gaíva MAM. Atenção odontológica para bebês: percepção de um grupo de mães. J Bras Odontoped Odonto Bebe. 2001; 4(22):485-489.

3. Peres SHCS, Cardoso MTV, Garcez RMVB, Peres AS, Bastos JRM. Tratamento alternativo de controle da cárie dentária no período materno infantil. Rev Assoc Paul Cir Dent. 2001; 55(5):346-350.

4. Dos Santos Junior VE, Sousa RM, Oliveira MC, Caldas Junior AF, Rosenblatt A. Early childhood caries and its relationship with perinatal, socioeconomic and nutritional risks: a cross-sectional study. BMC Oral Health. 2014; $14: 47$.

5. Moimaz SAS, Fadel CB, Lolli LF, Garbin CAS, Garbin AJI, Saliba NA. Social aspects of dental caries in the context of mother-child pairs. J Appl Oral Sci. 2014; 22 (1): 73-8.

6. Piovesan C, Marquezan M, Kramer PF, Bönecker M, Ardenghi TM. Socioeconomic and clinical factors associated with caregivers' perceptions of children's oral health in Brazil. Community Dent Oral Epidemiol 2011; 39(3):260-267.

7. Hayes A, Azarpazhooh A, Dempster L, Ravaghi V, Quiñonez C. Time loss due to dental problems and treatment in the Canadian population: analysis of a nationwide cross sectional survey. BMC Oral Health 2013; 13:17.

8. Stephen A, Krishnan R, Ramesh M, Kumar VS. Prevalence of early childhood caries and its risk factors in 18-72 month old children in Salem, Tamil Nadu. J Int Soc Prev Community Dent. 2015; 5(2): 95-102.

9. Bendo CB, Paiva SM, Abreu MH, Figueiredo LD, Vale MP. Impact of traumatic dental injuries among adolescents on family's quality of life: a populationbased study Int J Paediatr Dent 2014; 24 (5): 387396.
10. Massoni ACLT, Ferreira JMS, Silva FDSCM, Carvalho LFPC, Duarte RC. Conhecimento de Gestantes sobre a Saúde Bucal dos Bebês. R Bras Ci Saúde. 2009; 13(1):41-47.

11. Cruz LD, Novais SMA, Grinfeld S, Menezes JPL. Avaliação do conhecimento dos pais e condição de saúde bucal de bebês de 3 a 36 meses. ClipeOdontoUNITAU. 2010; 2(1): 2-9.

12. Farias $A Q$, Costa $C T$, Caminha RMC, Macedo DPC. Análise de conhecimentos e prática das mães sobre a saúde bucal de seus filhos na faixa etária de 0 a 6 anos do município de Casinhas/PE. Odontol Clín-Cient. 2012; 11(3); 243-245

13. Garbin CAS, Sumida DH, Santos RR, Chehoud Kamoimaz SAS. Saúde coletiva: promoção de saúde bucal na gravidez. Rev Odontol UNESP 2011; 40(4): 161-165.

14. Tiano AVP, Moimaz SAS, Saliba O, Saliba NA. Dental caries prevalence in children up to 36 months of age attending daycare centers in municipalities with different water fluoride contente. J Appl Oral Sci. 2009; 17(1): 39-44.

15. Cruz AAG, Gadelha CGF, Cavalcanti AL, Medeiros PFV. Percepção materna sobre a higiene bucal de bebês: Um estudo no hospital Alcides Carneiro, Campina Grande - PB. Pesq Bras Odontoped Clín Integr 2004; 4(3):185-189.

16. Hanna LMO, Nogueira AJS, Honda VYS. Percepção das gestantes sobre a atençãoodontológica precoce nos bebês. RGO. 2007;55(3): 271-274.

17. Guiotoku CM, Guiotoku SK. Conhecimento e percepção de mães da Unidade de Saúde Vila Verde em Curitiba PR em relação à higiene bucal de seus bebês. Revista Gestão \& Saúde. 2010; 1(2): 27-36.

18. Walter LRF, Ferelle A, Issao M. Odontologia para o Bebê: Odontopediatria do nascimento aos 3 anos. São Paulo: Artes Médicas; 1996.

19. Faustino-Silva DD, Ritter F, Nascimento IM, Fontanive PVN, Persici S, Rossoni E. Cuidados em saúde bucal na primeira infância: percepções e conhecimentos de pais ou responsáveis de crianças em um centro de saúde de Porto Alegre, RS. Rev. Odonto Ciênc. 2008;23(4):375-379. 
20. Bressane LB, Costa LNBS, Vieira JMR, Rebelo MAB Oral health conditions among pregnant women attended to at a health care center in Manaus, Amazonas, Brazil. Rev Odonto Cienc 2011;26(4):291-296.

21. Lacerda VR, Pontes ERJC, Queiroz CL. Relação entre senso de coerência materno, condições socioeconômicas e percepção da saúde bucal. Estudos de Psicologia 2012; 29(2): 203-8.
Correspondência

Julliana Cariry Palhano Freire

Av. Governador Argemiro de Figueiredo, 3826

Bessa, João Pessoa - PB.

CEP: $58037-030$ 